\title{
Monte Carlo simulations to replace film dosimetry in IMRT verification
}

\author{
Thomas Goetzfried $^{\mathrm{a}, *}$, Mark Rickhey ${ }^{\mathrm{b}}$, Marius Treutwein ${ }^{\mathrm{a}}$, \\ Oliver Koelbl ${ }^{\text {a }}$, Ludwig Bogner ${ }^{\mathrm{a}}$ \\ ${ }^{a}$ Department of Radiotherapy, Regensburg University Medical Center, D-93042 \\ Regensburg \\ ${ }^{\mathrm{b}}$ Institute of Radiotherapy and Oncology, Ingolstadt Medical Center, D-85049 \\ Ingolstadt
}

\begin{abstract}
Patient-specific verification of intensity-modulated radiation therapy (IMRT) plans can be done by dosimetric measurements or by independent dose or monitor unit calculations. The aim of this study was the clinical evaluation of IMRT verification based on a fast Monte Carlo (MC) program with regard to possible benefits compared to commonly used film dosimetry.

25 head-and-neck IMRT plans were recalculated by a pencil beam based treatment planning system (TPS) using an appropriate quality assurance (QA) phantom. All plans were verified both by film and diode dosimetry and compared to MC simulations. The irradiated films, the results of diode measurements and the computed dose distributions were evaluated, and the data were compared on the basis of gamma maps and dose-difference histograms.

Average deviations in the high-dose region between diode measurements and point dose calculations performed with the TPS and MC program were $0.7 \pm 2.7 \%$ and
\end{abstract}


$1.2 \pm 3.1 \%$, respectively. For film measurements, the mean gamma values with $3 \%$ dose difference and $3 \mathrm{~mm}$ distance-to-agreement were $0.74 \pm 0.28$ (TPS as reference) with dose deviations up to $10 \%$. Corresponding values were significantly reduced to $0.34 \pm 0.09$ for MC dose calculation. The total time needed for both verification procedures is comparable, however, by far less labor intensive in the case of $\mathrm{MC}$ simulations.

The presented study showed that independent dose calculation verification of IMRT plans with a fast MC program has the potential to eclipse film dosimetry more and more in the near future. Thus, the linac-specific QA part will necessarily become more important. In combination with MC simulations and due to the simple set-up, point-dose measurements for dosimetric plausibility checks are recommended at least in the IMRT introduction phase.

Key words: IMRT, Monte Carlo simulation, film dosimetry, QA

\section{Introduction}

The intensity-modulated radiotherapy (IMRT) technique is currently the most advanced form of conformal radiotherapy and holds great promise for improving radiotherapy both through increased tumor control probability and decreased treatment toxicity. As with all treatment modalities, verification and characterization measurements are required before clinically implementing IMRT. Moreover, it is apparent that comprehensive quality assurance (QA) is essential for IMRT due to the non-intuitive nature of treatment planning. Rec-

\footnotetext{
* Corresponding author.

Email address: thomas.goetzfried@klinik.uni-regensburg.de (Thomas Goetzfried).
} 
ommendations and guidelines for determining the appropriate use of IMRT, beginning an IMRT program, and maintaining the adequate QA to safely use IMRT are given in recently published and national regulatory reports [1-4]. Each institution's QA program should be designed to encompass the entire IMRT process, i.e. commissioning and validation of the dose calculation algorithm, data transfer from the treatment planning system (TPS) to the linac verify and record (V\&R) system, and the delivery process itself. After gaining experience, the IMRT QA is usually subdivided into a multileaf collimator (MLC)/linac-specific QA part and a patient-specific QA part. Both parts are parallel procedures and do not interfere directly [5]. When going this way, patient-specific verification of IMRT plans can be done by dosimetric measurements or by independent dose or MU calculations.

Dosimetric measurements typically comprise two types of checks for pre-treatment QA: single-beam verification and total-plan verification. In both cases, the patient's IMRT plan is transferred to a phantom and calculations are done at the points or depths of interest. Due to the characteristics of IMRT dose distributions including complex 3D treatment volume geometries, the precise characterization of these distributions using only point dosimeters is not practical. Mainly a comparison of two-dimensional calculated and measured data is performed. As a two-dimensional integrating dosimetry medium, radiographic films (an EDR-2 in particular) [6,7] or less commonly, radiochromic films [8] are nearly ideal for such measurements due to their very good resolution. Nevertheless, a multitude of alternative methods for patient dose validation, e.g. large scale 2D ion chamber or diode arrays as well as flat-panel electronic portal imaging devices (EPID) have been studied [9-11]. However, perhaps because of its familiarity and apparent simplicity, radiographic film remains one 
of the most common means for validating patient IMRT treatments through dose measurements. The use of radiographic films for dosimetry is described in detail in the AAPM Report 69 [12].

Methods based on Monte Carlo (MC) algorithms have been used for external beam radiotherapy dose calculation [13]. It is well accepted that MC is the most accurate dose calculation method, since it can precisely model realistic radiation transport through the accelerator treatment head, the MLC and the patient anatomy. Accordingly, $\mathrm{MC}$ has been regarded as a powerful tool to obtain the accurate dose distributions in the (heterogeneous) patient. Efforts have been made to implement MC in clinical treatment planning and plan verification [14], and suitable TPS are currently put on the market. There have been several fast MC codes developed, such as VMC/XVMC [15,16], DPM [17], MCV [18] or MCDOSE [19]. These codes employ a variety of variance reduction techniques and achieve reduced CPU time compared to ordinary EGSnrc calculations. Prior to clinical use, however, the calculation method should be commissioned against phantom measurements and the treatment planning system algorithm.

The aim of this work was to investigate potential advantages of replacing the quasi-3D verification with EDR-2 film dosimetry in (hybrid) phantoms by MC dose calculation. Within the present study, 25 head-and-neck IMRT plans have been evaluated both by experimental methods and by MC simulations. The evaluations have been performed with regard to the time investment for both procedures as well as the accuracy compared to the applied algorithm of the TPS. 


\section{Methods and materials}

\subsection{IMRT treatment plans}

Plans for 25 patients with head-and-neck cancer receiving step-and-shoot IMRT with a Siemens Primus ${ }^{\circledR}$ linear accelerator $(1 \mathrm{~cm}$ projected leaf width at the isocenter distance and $6 \mathrm{MV}$ photons) were used in the study. IMRT plans were based on a uniformly distributed seven-field arrangement except for two plans which had five fields, and were optimized with Oncentra ${ }^{\circledR}$ MasterPlan (v1.5, Nucletron). The number of segments per beam varied between 4 and 15 with a total number per plan between 48 and 80 and a minimum field size for one segment of $4 \mathrm{~cm}^{2}$. The total number of monitor units applied per plan were between 470 and 1287 with an average of 844 .

\subsection{Monte Carlo simulations}

The Monte Carlo simulations were performed using XVMC [20], into which the VEF model [21] is implemented in conjunction with geometry parameters for the patient-dependent portion of the treatment head (MLC and jaws). Since the commissioning procedure of this model is based on standard measurements in air but only on one depth dose in water for a reference field size, additional measurements in water were carried out to verify the model and its parameters. Percentage depth dose curves for standard field sizes and beam profiles in various depths were compared [22]. As an example, Figure 1 represents measured and calculated depth dose curves and profiles in water for a $6 \mathrm{MV} 5 \times 5 \mathrm{~cm}^{2}$ photon beam of a Siemens Primus ${ }^{\circledR}$ linac. The reference dose 
calibration is also consistent within $1 \%$. Only minor differences were found in output factor measurements (up to $5 \%$ for small fields).

XVMC/VEFM was used for forward calculations of all beam segments, as obtained by the pencil beam based IMRT treatment plans and transferred via DICOM-RT. The workflow has been previously described in detail [23]. The dose calculations were performed with up to $128 \times 128$ voxels (per slice). The size of a voxel varied from $0.2 \mathrm{~cm}$ in transversal plane to $0.5 \mathrm{~cm}$ in craniocaudal direction. The $1 \sigma$ statistical uncertainty in the dose was chosen to be $1-2 \%$ of the dose maximum $D_{\max }$.

\subsection{Verification procedures}

After an IMRT treatment plan was accepted, it was recalculated using identical beam settings but replacing the patient with a cylindrical polyethylene (PE) phantom (diameter $20 \mathrm{~cm}$, height $20 \mathrm{~cm}$ ). Such a phantom is easily accessible to dosimetry by radiographic films and point measurements [7]. It is possible to mark the coordinate system onto the films with a guided needle for data correlation and evaluation with IMRT software (PTW-VeriSoft ${ }^{\circledR}$ v3.0). Similar to the procedure of Ref. [24], a seven-step dose calibration $(0.5, \ldots$, $4.0 \mathrm{~Gy}$ ) with field sizes of $10 \times 3 \mathrm{~cm}^{2}$ at a depth of $10 \mathrm{~cm}$ of RW3 was applied within one automatic sequence of the V\&R system (Primeview ${ }^{\mathrm{TM}} \&$ Lantis $^{\circledR}$, Siemens) that exposes all fields to one film. As film and/or film processing changes with time, it is important to integrate the film calibration procedure into each film dosimetric investigation. The calibration procedure includes a non-linear fit of the optical density vs. dose data. An approach without the necessity of calibrating films before each IMRT verification measurement is 
reported in Ref. [25] by introducing normalized sensitometric curves and thus verifying IMRT plans in a relative manner. Although the stability of these curves with time and different film batches is very good, the authors recommend to check the shape of the sensitometric curve at regular intervals as part of the QA program, especially when new film batches or chemicals are used.

Additional determination of the absolute dose in a dose reference point was done using a semiconductor diode (PTW-60008) with an effective measuring volume of $1.1 \mathrm{~mm}$ in diameter and $2.5 \mu \mathrm{m}$ in thickness. For diode calibration the above mentioned cylindrical PE phantom along with a $0.3 \mathrm{cc}$ rigid stem ionization chamber (PTW-30016) as reference detector were used. The calibration factor was determined in IMRT reference conditions $($ SSD $90 \mathrm{~cm}$, field size of $5 \times 5 \mathrm{~cm}^{2}$ at isocenter and gantry angle of $0^{\circ}$ ). Due to the similarity of IMRT verification and calibration situations, no additional correction factors needed to be measured. The patient plan was copied onto the verification phantom in such a way that the diode was located in a high-dose region with no or just a small dose gradient $\left(<6 \% \mathrm{~cm}^{-1}\right)$. The procedure was repeated for all 25 IMRT plans included in this study. The absolute dose value was used to correct the film dose data in order to yield absolute 2D dose distributions.

For comparison with MC simulations, the dose distribution obtained from forward calculation was imported into the VeriSoft ${ }^{\circledR}$ software. Imported planes, i.e. dose distributions, are (automatically) resampled to a common pixel spacing of $0.5 \mathrm{~mm}$. On the basis of dose profiles and gamma maps [26], dose distributions of film dosimetry and MC simulations, respectively, vs. TPS dose calculation have been analyzed. Furthermore, difference matrices on a pixelby-pixel basis have been evaluated. Since this method is less helpful, if inhomogeneous dose distributions have to be examined, we focused on the high-dose 
region, i.e. between $1 \mathrm{~Gy}$ and $D_{\max }$, in a transversal plane in proximity to the isocenter of the phantom.

\section{Results}

The IMRT phantom plans were verified with EDR-2 type films, and forwardcalculation was carried out with the MC program. Average deviations in the high-dose region between diode measurements and point dose calculations performed with the TPS and MC program were $0.7 \pm 2.7 \%$ and $1.2 \pm 3.1 \%$, respectively. These deviations correspond to $1.2 \pm 4.8 \mathrm{cGy}$ and $2.0 \pm 5.0 \mathrm{cGy}$

per fraction. Film and MC dose distributions were then compared using the gamma evaluation method with $3 \%$ (local) dose difference and $3 \mathrm{~mm}$ distanceto-agreement as acceptance criteria. For this purpose, dose distributions obtained through the PB algorithm of the TPS were used as reference, as this algorithm is still the most frequently applied in clinical practice. The mean gamma values were $0.74 \pm 0.28$ and $0.34 \pm 0.09$ for film dosimetry and MC dose calculation, respectively, and $28.1 \pm 20.3 \%$ and $3.5 \pm 4.5 \%$ of all dose points were larger than 1. The latter values reduce to $6.3 \pm 5.8 \%$ and $1.2 \pm 1.8 \%$, respectively, if only the high-dose region, i.e. between $1 \mathrm{~Gy}$ and $D_{\max }$, is considered.

Figure 2 shows typical results of line dose calculations and measurements for one representative case. The dose profiles were calculated in a plane close to the isocenter and covered points up to $8 \mathrm{~cm}$ from the center of the phantom. In the inset, the high-dose region is bordered by a dash-dotted line representing the $1 \mathrm{~Gy}$ isodose. The deviations of film and MC calculation in the high-dose region compared to the $\mathrm{PB}$ calculated IMRT plan are visualized in histograms 
of the differences on a pixel-by-pixel basis (Figure 3). The results demonstrate a pronounced broadening for film measurement data up to $10 \%$ with mean $D$ of $-1.4 \%$ and $1 \sigma$ of $2.3 \%$ (relating to the prescribed dose of $2.0 \mathrm{~Gy}$ ). However, as mentioned above, in average only about $6 \%$ of all dose values (in the highdose region) fail the gamma index criteria $(3 \% / 3 \mathrm{~mm})$. For this reason, the film broadening may be attributed mainly to a small error in positioning the phantom on the linac couch or in placing the film within the phantom. For MC simulations, the standard deviation of $1.1 \%$ or $2.2 \mathrm{cGy}$ is significantly smaller.

The key histogram parameters for all 25 IMRT plans are illustrated in Figure 4. Except for two film verification measurements, the mean dose deviations remain within a confidence limit of $5 \%$ proposed for treatment plan acceptance [24]. Additionally, the highlighted region in the figure marks the range for the MC dose calculation results. Table 1 summarizes absolute and relative dose deviations of MC simulations and film dosimetry. The standard deviation between TPS PB calculations and film measurements, which reflect the variance of the 25 plans, was around 6 cGy for the mean dose values in the high-dose area, whereas for MC calculations the deviation was less than 2 cGy. In analogy, the corresponding key histogram parameters based on the comparison between MC dose calculation and film dosimetry can be deduced yielding to an average deviation of $1.7 \%$ (relating to the prescribed dose) for the mean dose value with a variance of $\sigma^{2}=0.001$.

Figure 5 demonstrates the total time needed for dosimetry with point detectors or films as well as for an independent dose calculation with XVMC/VEFM. The time for the individual steps of each procedure is based on the present verification study of 25 head-and-neck IMRT plans. The MC calculation required about $1-3 \mathrm{~h}$ on a single Pentium IV $2.4 \mathrm{GHz}$ PC depending on the 
number of segments and their sizes. While the average measuring time at the linac for both experimental methods was about 45 minutes each, film dosimetry was more comprehensive, doubling the total time compared to point-dose measurements.

\section{Discussion}

QA in IMRT is mainly founded on quantitative comparisons between computed and/or measured dose distributions. Current work on verification dose calculations for QA purposes focused on a comparison of the commonly practiced film dosimetry with an independent dose calculation by means of MC methods. Differences between measurement and calculation are principally caused by an error in planning, positioning, delivery or measurement technique. An agreement between the two distributions, on the contrary, is in itself not a proof of satisfying quality. Admittedly, the distributions that are compared may both contain uncertainty or bias, in such way that an agreement may be reached by chance. This consideration may serve as an argument to include many degrees of freedom, i.e., many measuring points, in the comparison. This means that comparing dose distributions is better than comparing doses measured in a limited set of points. Although a multitude of methods can be used for patient-specific dose based QA, individual treatment plans must be checked using time-effective and simple methods, realizing that this QA workload is proportional to the number of patients.

Regarding film dosimetry, there are several challenges in obtaining high-quality dosimetric results, namely, the dependence of optical density on photon energy, field size, depth, film batch sensitivity differences, film orientation, processing 
conditions, and scanner performance $[7,12]$. Based on film measurement results obtained in this study, an aimed confidence limit of $3 \%$ dose deviation (with respect to the prescribed dose) or 6 cGy, as suggested in Ref. [27], was only fulfilled for 16 out of 25 IMRT cases in the high-dose region. A significant lower uncertainty and dose deviation of less than $3 \%$ for all plans compared to the PB based TPS could be achieved when applying verification calculations with XVMC/VEFM; however, there were still slight differences. It is striking that the TPS with its PB algorithm had a tendency to overestimate the dose in the high dose area calculated with the MC program. These deviations may be attributed to computational uncertainty of the PB algorithm and to limitations in the accuracy of calculations at field edges and out of field [28]. In addition, discrepancies in output factor measurements for small fields up to $5 \%$ for $\mathrm{MC}$, and similar accuracy for $\mathrm{PB}$ calculations in a water phantom might be taken into consideration. Same uncertainties have to be kept in mind when comparing MC calculated dose distribution to film measurements. In addition to the challenge in context to correct film dosimetry, these difficulties contribute to the observed deviations between MC and film dose distributions. Comparing MC and TPS PB with film dosimetry, the variance in dose difference $\left(\sigma^{2}=0.001\right)$, however, is very similar for both comparisons. As commonly known, Monte Carlo simulations are proven to be the gold standard in radiation dose calculation. Nevertheless, there are a few aspects that need careful consideration.

While the total time for MC simulations is comparable to film dosimetry, no extra time on the linac is required. Moreover, the manpower requirements for MC dose calculation are reduced to the DICOM file export from the TPS and import into the MC program. Verification calculations though will not 
detect errors in the IMRT delivery process. For this reason, independent calculations in addition to measurements of single dose points should be part of a rigorous control of the dose delivery system. However, it is to be noted that there are also other promising approaches, e.g. the log file based Monte Carlo simulations [29]. The authors suggest to use this method as an initial IMRT QA procedure to screen the plans, and the measurement based methods as a second procedure only when necessary.

Independent dose calculations (and dosimetric measurements) are typically performed in a homogeneous verification phantom. Hence, the influence of inhomogeneities present in the patient is not taken into account. Several studies, however, have shown the limitations of PB algorithms in heterogeneous media [30,31]. The PB algorithm produces large errors for the dose in the vicinity of interfaces and within low-density regions. In contrast, XVMC/VEFM calculations are very close to measurements even in the low-density area [31], which may be important when calculating dose to inhomogeneous volumes such as the head-and-neck region studied in this work. Further research is needed to investigate these effects on the dose distribution in the (heterogeneous) patient and to make use of MC simulations as verification tool based on the patient CT data set.

\section{Conclusion}

One should keep clearly in mind that dosimetric measurements are timeconsuming and only verify the dose in a phantom geometry. Discrepancies between planar measurements and calculations may be difficult to interpret in terms of the clinical significance on a patient-by-patient basis. Independent 
monitor unit or dose calculations offer an alternative to dose measurements. Monte Carlo is becoming fast enough to be used in clinics with the capability of accurate dose computations [32-35]. Once a calculation method is properly verified, significant time can be saved when compared to measurements. However, additional QA checks are needed to verify accurate transfer of patient data and accurate delivery. Thus, a more stringent machine and MLC QA needs to be considered. In this context, deficiency inevitably lead to differences between calculated and measured dose distributions. As mentioned in the introduction, dosimetric plausibility checks in terms of point-dose measurements are considered appropriate. Dose calculation inaccuracies due to use of approximation algorithms like pencil beam or collapsed cone can potentially be detected by MC simulations of the treatment plan in the patient itself instead of in a homogeneous phantom. The 3D dose verification procedure dealing with this issue is currently under investigation and will be reported.

\section{Acknowledgements}

This work was financially supported by the Bavarian Ministry for Environment, Health and Consumer. The Monte Carlo code XVMC was kindly provided by M. Fippel.

\section{References}

[1] Ezzell GA, Galvin JM, Low D, Palta JR, Rosen I, Sharpe MB, et al. Guidance document on delivery, treatment planning, and clinical implementation of 
IMRT: report of the IMRT Subcommittee of the AAPM Radiation Therapy Committee. Med Phys 2003;30(8):2089-115.

[2] Galvin JM, Ezzell G, Eisbrauch A, Yu C, Butler B, Xiao Y, et al. Implementing IMRT in clinical practice: a joint document of the American Society for Therapeutic Radiology and Oncology and the American Association of Physicists in Medicine. Int J Radiat Oncol Biol Phys 2004;58(5):1616-34.

[3] Williams PC. IMRT: delivery techniques and quality assurance. Br J Radiol 2003;76(911):766-76.

[4] DIN ('Deutsche Industrie-Norm') 6875-3, Ausgabe 2008-3 (Norm), spezielle Bestrahlungseinrichtungen - Teil 3: Fluenzmodulierte Strahlentherapie Kennmerkmale, Prüfmethoden und Regeln für den klinischen Einsatz. Normenausschuss Radiologie. Berlin, Beuth; 2008.

[5] Wagter CD. 10. In: Bortfeld T, Schmidt-Ullrich R, Neve WD, Wazer DE, editors. Image-Guided IMRT. 13. Springer-Verlag; 2006, p. 117-28.

[6] Childress NL, Salehpour M, Dong L, Bloch C, White RA, Rosen II. Dosimetric accuracy of Kodak EDR2 film for IMRT verifications. Med Phys $2005 ; 32(2): 539-48$.

[7] Bogner L, Scherer J, Treutwein M, Hartmann M, Gum F, Amediek A. Verification of IMRT: techniques and problems. Strahlenther Onkol 2004;180(6):340-50.

[8] Todorovic M, Fischer M, Cremers F, Thom E, Schmidt R. Evaluation of GafChromic EBT prototype B for external beam dose verification. Med Phys $2006 ; 33(5): 1321-28$.

[9] Winkler P, Hefner A, Georg D. Implementation and validation of portal dosimetry with an amorphous silicon EPID in the energy range from 6 to 25 MV. Phys Med Biol 2007;52(15):N355-65. 
[10] Esch AV, Depuydt T, Huyskens DP. The use of an aSi-based EPID for routine absolute dosimetric pre-treatment verification of dynamic IMRT fields. Radiother Oncol 2004;71(2):223-34.

[11] Herzen J, Todorovic M, Cremers F, Platz V, Albers D, Bartels A, et al. Dosimetric evaluation of a $2 \mathrm{D}$ pixel ionization chamber for implementation in clinical routine. Phys Med Biol 2007;52(4):1197-208.

[12] Pai S, Das IJ, Dempsey JF, Lam KL, Losasso TJ, Olch AJ, et al. TG-69: radiographic film for megavoltage beam dosimetry. Med Phys 2007;34(6):222858.

[13] Mackie TR. Applications of the Monte Carlo method in radiotherapy. In: K Kase BB, Attix FH, editors. The Dosimetry of Ionizing Radiation. vol. 3. New York: Academic Press; 1990, p. 541-620.

[14] Ma CM, Pawlicki T, Jiang SB, Li JS, Deng J, Mok E, et al. Monte Carlo verification of IMRT dose distributions from a commercial treatment planning optimization system. Phys Med Biol 2000;45(9):2483-95.

[15] Kawrakow I, Fippel M, Friedrich K. 3D electron dose calculation using a Voxel based Monte Carlo algorithm (VMC). Med Phys 1996;23(4):445-57.

[16] Fippel M, Laub W, Huber B, Nüsslin F. Experimental investigation of a fast Monte Carlo photon beam dose calculation algorithm. Phys Med Biol 1999;44(12):3039-54.

[17] Sempau J, Wilderman SJ, Bielajew AF. DPM, a fast, accurate Monte Carlo code optimized for photon and electron radiotherapy treatment planning dose calculations. Phys Med Biol 2000;45(8):2263-91.

[18] Siebers JV, Keall PJ, Kim JO, Mohan R. Performance benchmarks of the MCV Monte Carlo system. In: Schlegel W, Bortfeld T, editors. Proc. 13th Int. Conf. 
on the Use of Computers in Radiation Therapy (ICCR) (Heidelberg, Germany). Berlin: Springer; 2000, p. 129-31.

[19] Ma CM, Li JS, Pawlicki T, Jiang SB, Deng J, Lee MC, et al. A Monte Carlo dose calculation tool for radiotherapy treatment planning. Phys Med Biol 2002;47(10):1671-89.

[20] Fippel M. Fast Monte Carlo dose calculation for photon beams based on the VMC electron algorithm. Med Phys 1999;26(8):1466-75.

[21] Fippel M, Haryanto F, Dohm O, Nüsslin F, Kriesen S. A virtual photon energy fluence model for Monte Carlo dose calculation. Med Phys 2003;30(3):301-11.

[22] Rickhey M. Entwicklung einer biologisch adaptierten intensitätsmodulierten Strahlentherapieplanung auf der Basis molekularbiologischer Bildgebungsverfahren. PhD Thesis 2008.

[23] Bogner L, Hartmann M, Rickhey M, Moravek Z. Application of an inverse kernel concept to Monte Carlo based IMRT. Med Phys 2006;33(12):4749-57.

[24] Rhein B, Häring P, Debus J, Schlegel W. Dosimetrische Verifikation von IMRTGesamtplänen am Deutschen Krebsforschungszentrum Heidelberg. Z Med Phys $2002 ; 12: 122-33$.

[25] Georg D, Kroupa B, Winkler P, Pötter, R. Normalized sensitometric curves for the verification of hybrid IMRT treatment plans with multiple energies. Med Phys 2003;30(6):1142-50.

[26] Low DA, Harms WB, Mutic S, Purdy JA. A technique for the quantitative evaluation of dose distributions. Med Phys 1998;25(5):656-61.

[27] Georg D, Stock M, Kroupa B, Olofsson J, Nyholm T, Ahnesjö A, et al. Patientspecific IMRT verification using independent fluence-based dose calculation software: experimental benchmarking and initial clinical experience. Phys Med Biol 2007;52(16):4981-92. 
[28] Schwarz M, Bos LJ, Mijnheer BJ, Lebesque JV, Damen EMF. Importance of accurate dose calculations outside segment edges in intensity modulated radiotherapy treatment planning. Radiother Oncol 2003;69(3):305-14.

[29] Luo W, Li J, Price RA, Chen L, Yang J, Fan J, et al. Monte Carlo based IMRT dose verification using MLC $\log$ files and $\mathrm{R} / \mathrm{V}$ outputs. Med Phys $2006 ; 33(7): 2557-64$.

[30] Ahnesjoe A, Aspradakis MM. Dose calculations for external photon beams in radiotherapy. Phys Med Biol 1999;44(11):R99-155.

[31] Krieger T, Sauer OA. Monte Carlo- versus pencil-beam-/collapsed-conedose calculation in a heterogeneous multi-layer phantom. Phys Med Biol $2005 ; 50(5): 859-68$.

[32] Laub W, Alber M, Birkner M, Nüsslin F. Monte Carlo dose computation for IMRT optimization. Phys Med Biol 2000;45(7):1741-54.

[33] Siebers JV, Lauterbach M, Tong S, Wu Q, Mohan R. Reducing dose calculation time for accurate iterative IMRT planning. Med Phys 2002;29(2):231-7.

[34] Jeraj R, Keall PJ, Siebers JV. The effect of dose calculation accuracy on inverse treatment planning. Phys Med Biol 2002;47(3):391-407.

[35] Hartmann M, Bogner L, Fippel M, Scherer J, Scherer S. IMCO(++)-a Monte Carlo based IMRT system. Z Med Phys 2002;12(2):97-108. 


\section{Table 1}

Mean and standard (absolute and relative) dose deviations in the high-dose region (between 1 Gy and $D_{\max }$ ) for 25 IMRT phantom plans. Results obtained with the pencil beam algorithm of the TPS were used as reference for comparison to film measurements and Monte Carlo calculations.

Absolute deviation (cGy) Relating to prescribed dose (\%)

\begin{tabular}{lll} 
Film & $0.3 \pm 5.7$ & $0.2 \pm 3.0$ \\
& & \\
$\mathrm{MC}$ & $-2.7 \pm 1.4$ & $-1.4 \pm 0.7$ \\
\hline
\end{tabular}




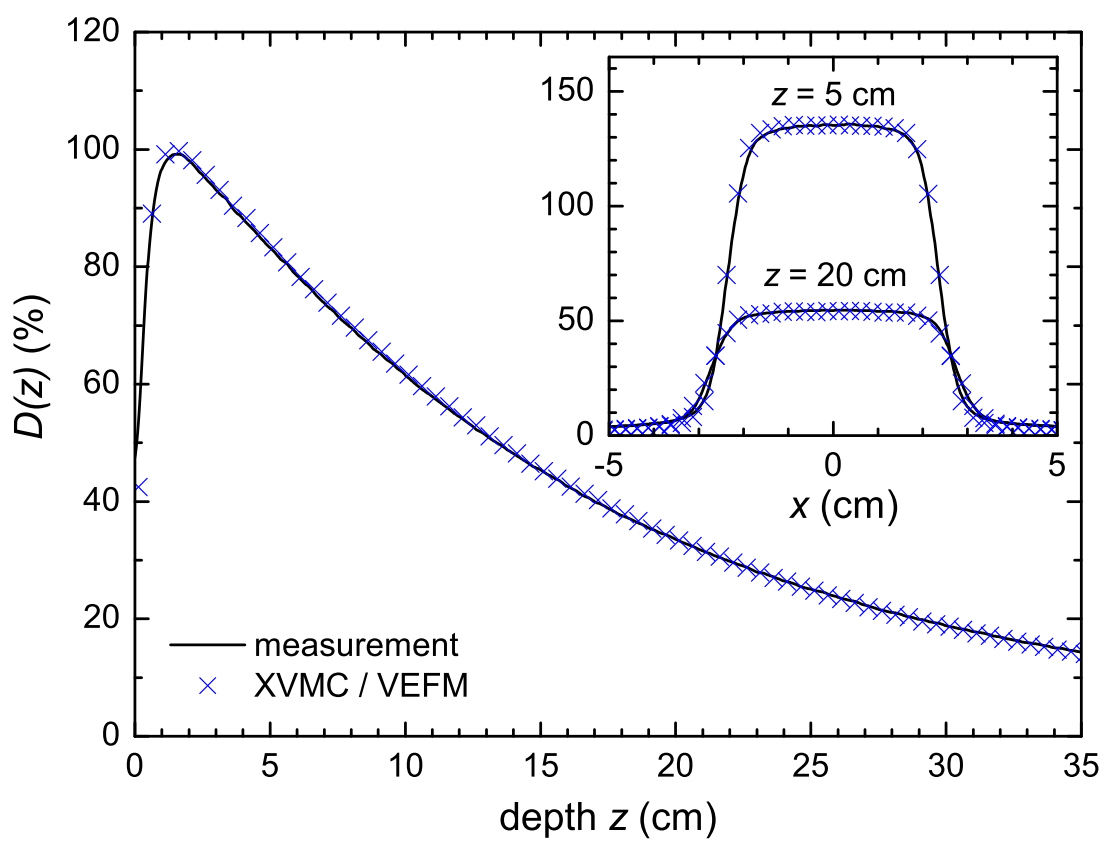

Fig. 1. Measured (solid lines) compared to calculated (symbols) depth dose curves in water for a $6 \mathrm{MV} 5 \times 5 \mathrm{~cm}^{2}$ photon beam of a Siemens Primus ${ }^{\circledR}$ linear accelerator. In the inset, the respective profiles in $x$ direction at 5 and $20 \mathrm{~cm}$ depths in water are shown. 


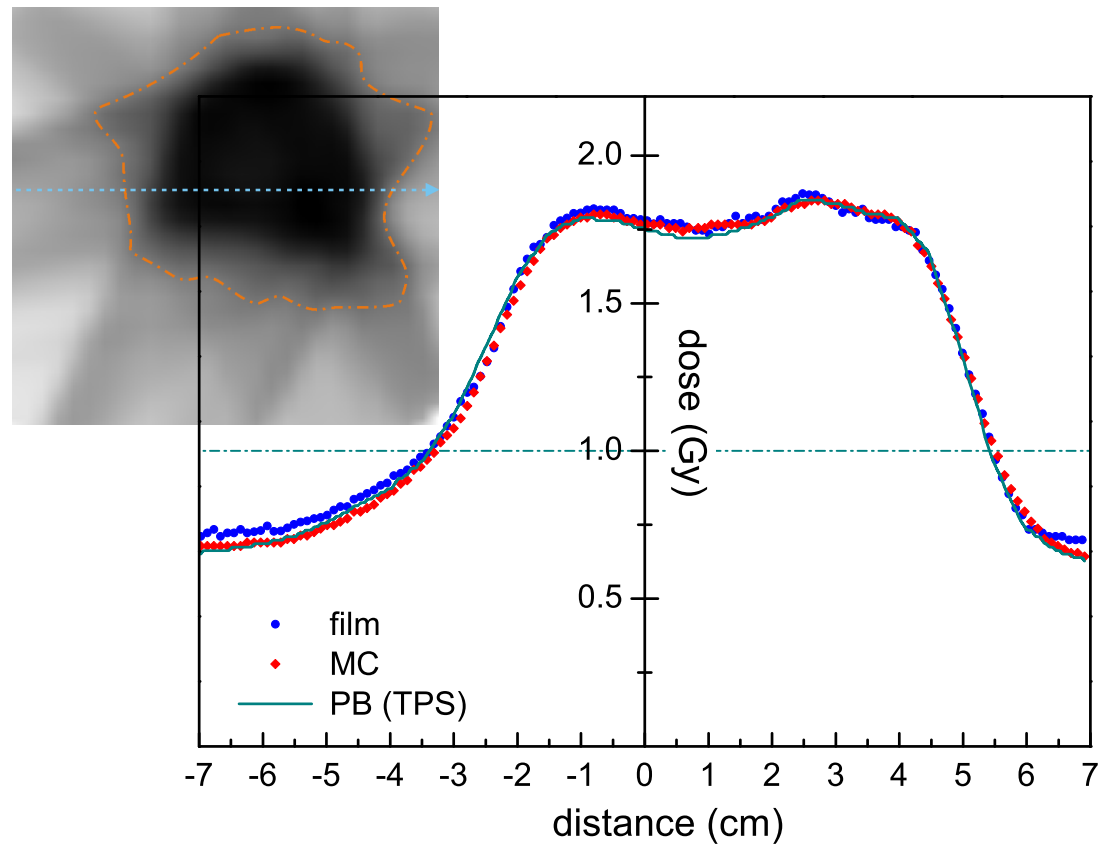

Fig. 2. Line dose profiles for a representative pencil beam based IMRT plan verified by film dosimetry and MC forward calculation. The inset illustrates the dose distribution and the position of the line verification. The dash-dotted lines (both figures) represent the lower limit of the high-dose region. 


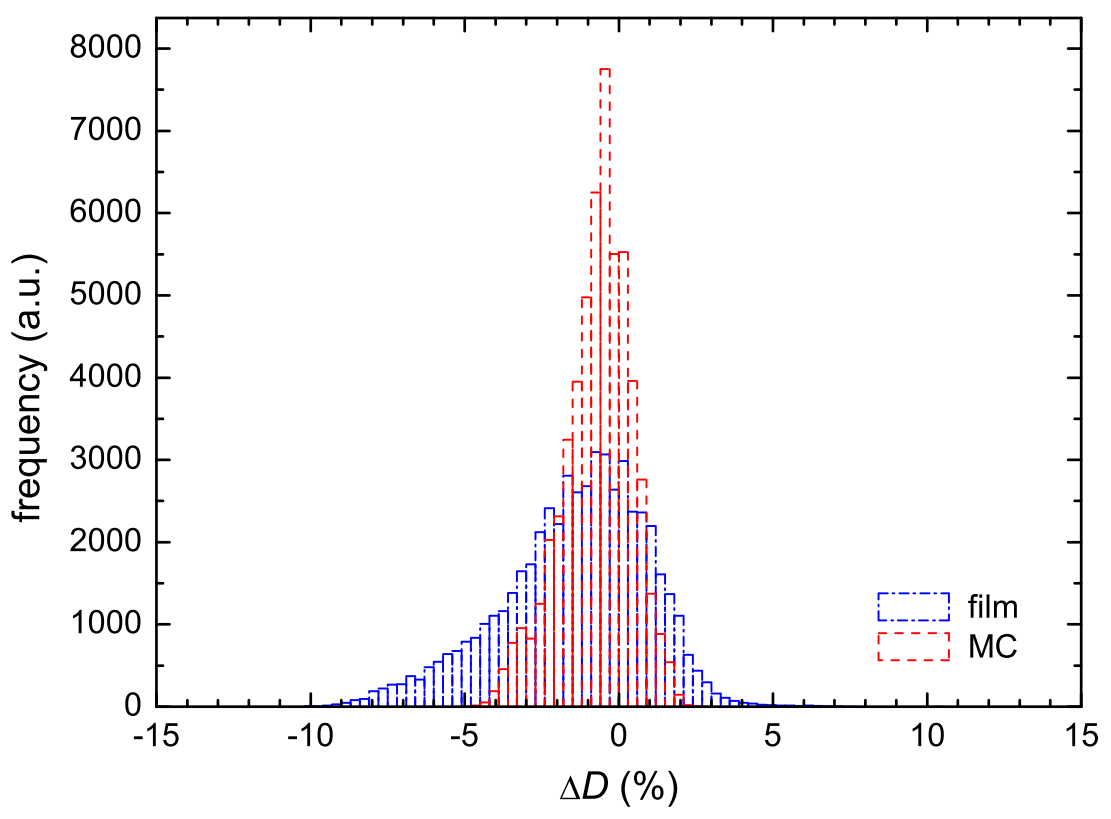

Fig. 3. Dose-difference histograms derived from the difference between film dosimetry and MC simulations, respectively, and the $\mathrm{PB}$ dose calculation performed with the treatment planning system (in the high-dose region). The dose difference $D_{(\text {film/MC) }}-D_{\mathrm{PB}}$ is normalized to the prescribed dose of the PB plan. 


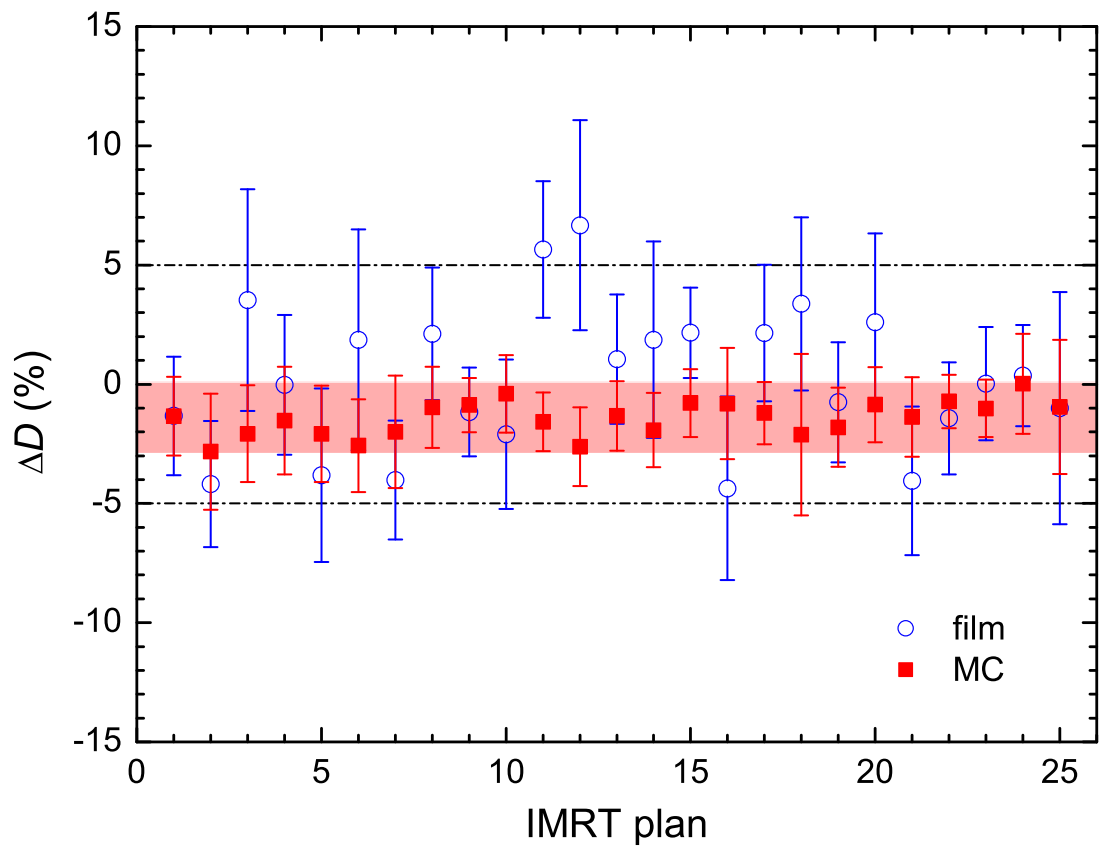

Fig. 4. Results of film verification measurements and MC forward calculations for all 25 IMRT plans, shown as mean dose values and respective standard deviation obtained from dose-difference histograms (see also Figure 3). The two dash-dotted lines denote a confidence limit of $5 \%$, the highlighted region marks the range for MC dose calculation results. 


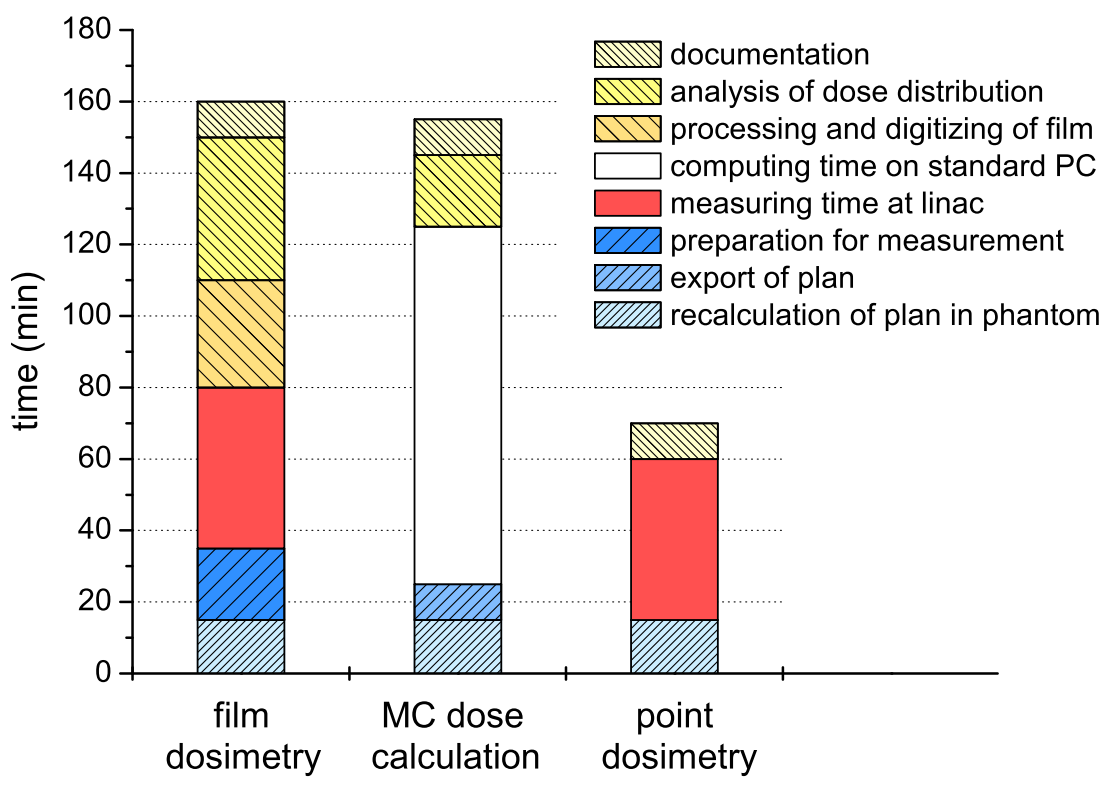

Fig. 5. Time need for dosimetry (point detector and film) and independent dose calculation of IMRT plans, including preparation and postprocessing. The time for the individual steps is based on the present verification study of 25 head-and-neck IMRT plans. 